\title{
Multiple Myeloma Patients Undergoing Carfilzomib: Development and Validation of a Risk Score for Cardiovascular Adverse Events Prediction
}

\author{
Anna Astarita ${ }^{1, *}$, Giulia Mingrone ${ }^{1}{ }^{\mathbb{D}}$, Lorenzo Airale ${ }^{1}$, Fabrizio Vallelonga ${ }^{1}$, Michele Covella ${ }^{1}$, \\ Cinzia Catarinella ${ }^{1}$, Marco Cesareo ${ }^{1}$, Giulia Bruno ${ }^{1}$, Dario Leone ${ }^{1}{ }^{1}$, Carlo Giordana ${ }^{1}$, Giusy Cetani ${ }^{2}$, \\ Marco Salvini ${ }^{2}$, Francesca Gay ${ }^{2}$, Sara Bringhen ${ }^{2}{ }^{\circledR}$, Franco Rabbia ${ }^{1}$, Franco Veglio ${ }^{1}$ and Alberto Milan ${ }^{1} \mathbb{C}$
}

1 Hypertension Unit, Department of Medical Sciences, Division of Internal Medicine, AO “Città della Salute e della Scienza" University Hospital, 10126 Turin, Italy; giulia.mingrone@edu.unito.it (G.M.); lorenzo.airale@unito.it (L.A.); fabrizio.vallelonga@unito.it (F.V.); mcovella@ausl.vda.it (M.C.); cinzia.catarinella@edu.unito.it (C.C.); marcoriccardo.cesareo@unito.it (M.C.); giulia.bruno87@gmail.com (G.B.); dgleone@live.it (D.L.); carlo.giordana@edu.unito.it (C.G.); franco.rabbia@libero.it (F.R.); franco.veglio@unito.it (F.V.); alberto.milan@gmail.com (A.M.)

2 Myeloma Unit, Department of Medical Sciences, Division of Hematology, AO “Città della Salute e della Scienza" University Hospital, 10126 Turin, Italy; g.cetani@gmail.com (G.C.); marcosalvini83@gmail.com (M.S.); francesca.gay@unito.it (F.G.); sarabringhen@yahoo.com (S.B.)

* Correspondence: anna.astarita@unito.it; Tel.: +39-011-633-6959

Citation: Astarita, A.; Mingrone, G.; Airale, L.; Vallelonga, F.; Covella, M.; Catarinella, C.; Cesareo, M.; Bruno, G.; Leone, D.; Giordana, C.; et al. Multiple Myeloma Patients Undergoing Carfilzomib: Development and Validation of a Risk Score for Cardiovascular Adverse Events Prediction. Cancers 2021, 13, 1631. https://doi.org/10.3390/cancers 13071631

Academic Editor: Nicola Amodio

Received: 18 February 2021

Accepted: 28 March 2021

Published: 1 April 2021

Publisher's Note: MDPI stays neutral with regard to jurisdictional claims in published maps and institutional affiliations.

Copyright: (c) 2021 by the authors. Licensee MDPI, Basel, Switzerland. This article is an open access article distributed under the terms and conditions of the Creative Commons Attribution (CC BY) license (https:// creativecommons.org/licenses/by/ $4.0 /)$.
Simple Summary: Despite the relationship between Carfilzomib (CFZ) therapy in multiple myeloma (MM) and cardiovascular adverse events (CVAEs), no specific validated protocols on cardiovascular risk assessment are available. In this prospective study, we investigated major predictors of CVAEs prior to starting CFZ, applying the European Myeloma Network management protocol (EMN). Five predictors were identified: office systolic blood pressure, 24-h blood pressure variability, left ventricular hypertrophy, pulse wave velocity value and global longitudinal strain. The resulting 'CVAEs risk score' defined a low- and a high-risk group (negative predicting value for the high-risk group of $90 \%$ ). 62 patients experienced one or more CVAEs: 17 major and 45 hypertension-related events. In conclusion, CVAEs are frequent and a specific management protocol is required. The EMN protocol and 'CFZ risk score' proved to be effective in estimating the baseline risk of CVAEs during CFZ therapy in MM patients, targeting the appropriate follow-up.

Abstract: Cardiovascular adverse events (CVAEs) are linked to Carfilzomib (CFZ) therapy in multiple myeloma (MM); however, no validated protocols on cardiovascular risk assessment are available. In this prospective study, the effectiveness of the European Myeloma Network protocol (EMN) in cardiovascular risk assessment was investigated, identifying major predictors of CVAEs. From January 2015 to March 2020, 116 MM patients who had indication for CFZ therapy underwent a baseline evaluation (including blood pressure measurements, echocardiography and arterial stiffness estimation) and were prospectively followed. The median age was $64.53 \pm 8.42$ years old, $56 \%$ male. Five baseline independent predictors of CVAEs were identified: office systolic blood pressure, 24-h blood pressure variability, left ventricular hypertrophy, pulse wave velocity value and global longitudinal strain. The resulting 'CVAEs risk score' distinguished a low- and a high-risk group, obtaining a negative predicting value for the high-risk group of $90 \%$. 52 patients $(44.9 \%)$ experienced one or more CVAEs: $17(14.7 \%)$ had major and $45(38.7 \%)$ had hypertension-related events. In conclusion, CVAEs are frequent and a specific management protocol is crucial. The EMN protocol and the risk score proved to be useful to estimate the baseline risk for CVAEs during CFZ therapy, allowing the identification of higher-risk patients.

Keywords: multiple myeloma; carfilzomib; cardiovascular adverse events; cardiovascular risk assessment; arterial hypertension; echocardiography; global longitudinal strain; pulse wave velocity 


\section{Introduction}

Carfilzomib (CFZ) is an irreversible second-generation proteasome inhibitor which has shown a significant improvement in survival rates in relapsed and/or refractory multiple myeloma (MM) [1]. Since its approval, several reports [2-4] highlighted a connection between use of CFZ and cardiovascular adverse events (CVAEs), due to an overlapping of risk factors, involving irreversible proteasome inhibitor cardiotoxicity and the widerange of MM-related comorbidities. Heart failure, arrhythmias, acute coronary syndromes, sudden cardiac death and hypertensive events have all been described [5,6]. A review and meta-analysis, including phase 1 to 3 prospective trials on CFZ therapy in MM patients, estimated an $18.1 \%$ incidence of CVAEs during CFZ, with a high frequency of hypertensive events and heart failure [7].

In spite of the increased potential for life-threatening CVAEs, no validated management protocols on cardiovascular risk assessment are available, making it challenging to assess the individual cardiovascular risk profile and to estimate the probability of CVAEs during CFZ therapy. Furthermore, the risk factors and real incidence of each type of CVAE is unknown, due to lack of 'real-life' studies. The key objective of this prospective study was to evaluate the effectiveness of the multimodality assessment protocol proposed by the Consensus Paper of the European Myeloma Network (EMN) [8] for the cardiovascular risk assessment of MM patients undergoing CFZ therapy. Major predictors of CVAEs were identified and a risk score prediction model for CVAEs was developed in order to identify patients at higher risk for cardiovascular events, and consequently with higher risk for discontinuation of CFZ therapy, who require a closer and tailored follow-up. The type and incidence of CVAEs were investigated.

\section{Materials and Methods}

This cohort prospective study was conducted at the third level Hypertension Unit and Centre for Cardiovascular diseases of 'Città della Salute e della Scienza' Hospital in Turin, Italy. Patients with MM who had indication for CFZ were consecutively enrolled between January 2015 and March 2020. Patients previously treated with CFZ, those affected by light chain cardiac amyloidosis (assessed by end-organ biopsy or cardiac magnetic resonance imaging) and those refusing informed consent were excluded.

\subsection{Baseline Assessment before Starting CFZ}

In accordance with the European Myeloma Network Protocol [8], before starting CFZ therapy, patients underwent a detailed baseline assessment, including cardiovascular history, physical examination, office blood pressure measurements, ambulatory blood pressure monitoring (ABPM), 12-leads ECG, trans-thoracic echocardiography with global longitudinal strain assessment (GLS) and estimation of arterial stiffness through pulse wave velocity (PWV). Office blood pressure (BP) was recorded by an automatic sphygmomanometer: three consecutives BP measurements 1-2 min apart (supine, standing, sitting) were performed and the mean value was used (Omron, M10-IT model; Omron Healthcare Co.; Kyoto, Japan) [9]. ABPM was performed with a 24-h recording (reading taken every 15 minutes throughout the day and every 30 minutes during the night), using a validated measuring device (Takeda TM2430; A\&D Company Ltd, Tokyo, Japan). The following measurements were extracted: systolic BP, diastolic BP, mean BP and standard deviation during the 24-hours, during the day and night, blood pressure variability (BPV) (derived from the average of night-time SD corrected for the respective duration of night), pulse pressure (difference between systolic BP-diastolic BP) and dipping value (derived using the formula (1-(SBP night/SBP day)) [9]. Trans-thoracic echocardiography was performed placing the patient in left lateral decubitus position (iE33 ultrasound machine, Philips Medical System, Andover, MA, USA). In parasternal long-axis view, left ventricular (LV) diameters and wall thickness were measured (deriving LV geometry through the Deveraux formula indexed to both body surface area and height elevated to 2.7). LV hypertrophy (LVH) was defined in presence of LV mass $\geq 115 \mathrm{~g} / \mathrm{m} 2(\geq 49 \mathrm{~g} / \mathrm{m} 2.7)$ and $\geq 95 \mathrm{~g} / \mathrm{m} 2(\geq 47 \mathrm{~g} / \mathrm{m} 2.7)$ in 
men and women, respectively. LV ejection fraction (LVEF) and LV volumes were assessed from the apical 4- and 2-chamber views indexed to body surface area (Simpson's Biplane technique) [10]. The LV diastolic function was assessed using the combination of TDI ( $\mathrm{e}^{\prime}$ septal and lateral anulus mitral waves), Pulsed Doppler (E and A mitral valve waves), tricuspid regurgitation peak velocity, left atrial volume (indexed to body surface area) and $\mathrm{E} / \mathrm{e}^{\prime}$ ratio (according to the current recommendations) [11]. The global longitudinal strain (GLS) was performed using the speckle tracking analysis system, using dedicated software (Automated Cardiac Motion Quantification, QLAB Cardiac Analysis, Philips, Andover, MA, USA). The GLS was computed offline from standard 2D images of the LV apical views (4-,3- 2- chamber), tracing the endocardium border by semi-automatic system with manual adjustment when needed, performed by standardized protocols [12]. Arterial stiffness estimation was performed through the carotid-femoral pulse wave velocity (PWV) using a validated device (Sphygmocor system Atcor Medical, Sydney, Australia). A single applanation tonometer was used to obtain and record carotid and femoral pulse waveforms, performed by expert operators. The PWV value was derived from the formula: distance covered by the pulse wave divided by the delay time (measured between the feet of the two waveforms). The mean of at least two PWV measurements (considering a standard deviation for each record $<10 \%$ ), were considered valid for the analysis, according to the current guidelines [13]. During the first visit, patients with either office or out-of-office blood pressure values in the high/normal range and those with arterial hypertension, according to the ESH recommendations [9], were advised to start anti-hypertensive treatment or to optimize the previous anti-hypertensive therapy in order to obtain blood pressure control before starting CFZ therapy. CFZ-based regimen, timing and dosing were decided by the hematologists.

\subsection{Follow-Up Assessments and CVAEs}

Follow-up assessments were performed every six months and/or at the time of any suspected CVAE. The type and the incidence of CVAEs were checked during the clinical evaluations, through periodic review of patients' electronic reports and by phone interviews. Patients were followed until the end of CFZ therapy. CVAEs were ranked into two subgroups: major CVAEs and arterial hypertension-related CVAEs. Specifically, we considered as 'major CVAE' all the events having a cardiovascular origin, excluding analysis which could not be expressively related to cardiovascular causes (CVAEs' definitions used are available in the Appendix A). CVAE events were graded according to the Common Terminology Criteria for Adverse Events version 5.0 (CTCAE 5.0) [14].

\subsection{Statistical Analysis}

Difference in baseline parameters between patients who experienced CVAEs and patients who did not experience CVAEs was investigated by the chi-square test/Fisher's exact test for categorical variables and by the unpaired $t$-test/Mann Whitney test for continuous variables, as appropriate. A two-sided $p$ value less than 0.05 was used as the level of statistical significance. The univariate binary logistic regressions were used to investigate the hazard ratio of each variable for CVAEs. The analysis was performed using a dedicated software (IBM SPSS Statistics, Version 22.0.0.0, IBM Corp., Armonk, NY, USA).

\subsection{CFZ CVAEs Risk Score Analysis}

The CFZ CVAEs Risk Score was developed according to the CHARMS Recommendations [15] and adheres to the TRIPOD Statement for Prediction Model [16]. The analysis was performed using a dedicated software (R: A Language and Environment for Statistical Computing, v.4.0.0 for Mac OSX, R Core Team, Vienna, Austria). Missing data (3.8\% of the 1276 total variables) were estimated by multiple imputations system as recommended $[15,17]$. Regression analysis was performed by two penalized models: the first, with the aim of reducing the set of covariates [18], and the second with the aim of building a risk predictor system, the 'CFZ CVAEs risk score'. The score was internally validated by bootstrapping 
according to the CHARMS Recommendations [15]: 1000 random samplings with replacement from the original sample were performed in order to assess the discrimination and the calibration of the score. Discrimination was calculated with $C$ statistic, and calibration was assessed using the calibration plot and the calibration slope. The optimal threshold was identified by imposing a sensibility value $\geq 90 \%$.

\section{Results}

\subsection{Baseline Characteristics and Cardiovascular Risk Factors}

Of 148 patients, 116 matched the inclusion criteria and they were enrolled (Figure 1 shows the flow-chart of the protocol). The baseline characteristics of the study sample are listed in Table 1. Tobacco use (50\%) and known arterial hypertension $(41.1 \%)$ were the most common cardiovascular $(\mathrm{CV})$ risk factors. According to office blood pressure values and/or ABPM, 51.7\% met the criteria for arterial hypertension, requiring the introduction of a new anti-hypertensive treatment or a modification of their previous therapy. The prevalence of asymptomatic hypertension-mediated organ damage (HMOD) was substantial: $20.7 \%$ had left ventricular hypertrophy at echocardiography evaluation, $20.7 \%$ had GLS impairment (defined as GLS $\leq 20 \%$ ) and $26.7 \%$ showed increased arterial stiffness $(\mathrm{PWV} \geq 9 \mathrm{~m} / \mathrm{s})$. The ABPM was available for 107 patients (nine patients had inappropriate recording/invalid result $<70 \%$ day/night measurements), the GLS assessment for 115 patients (one patient had echo images with poor quality) and PWV assessment for 103 patients (13 patients had less than two valid measurements due to tachycardia, extrasystoles, or technical difficulty due to overweight).

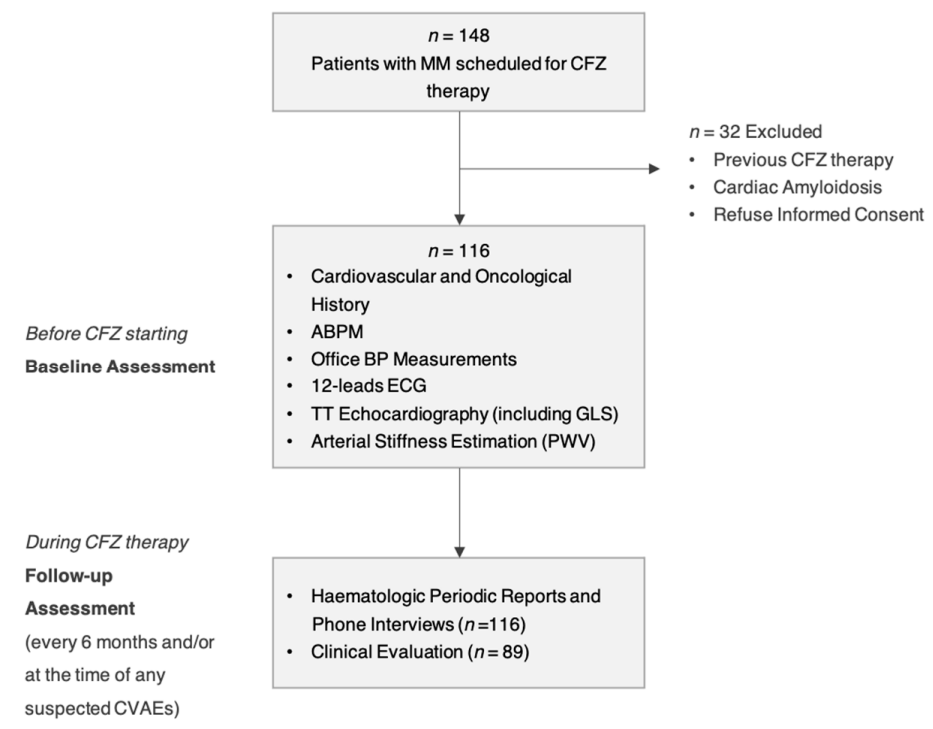

Figure 1. Flow-chart of the Study Protocol.

\subsection{CFZ Therapy Regimen: Timing and Dose}

The median duration of CFZ therapy was 8.6 months (0.1 to 52.8) for a median cumulative dose of 2781.9 (29.4-1323.5) $\mathrm{mg}$ of CFZ (computed at the end of the planned therapy or at the end of the study if CFZ was still ongoing). The most common regimen was a combined protocol with lenalidomide and dexamethasone (53 patients; 45.6\%) [19]. $79(86.1 \%)$ patients received up to $36 \mathrm{mg} / \mathrm{m} 2$ of CFZ dose per administration, $32(27.5 \%)$ up to $56 \mathrm{mg} / \mathrm{m} 2$ and three (2.6\%) up to $70 \mathrm{mg} / \mathrm{m} 2$, without differences observed in CVAEs between groups ( $p>0.05$ for all comparisons). At the end of the study, 89 patients $(76.2 \%)$ had discontinued CFZ therapy. Causes for discontinuation included disease progression $(n=64)$, patients' decision $(n=6)$, non-cardiovascular toxicity (renal, hematological, gastrointestinal) $(n=12)$ and $(n=7)$ cardiovascular toxicity $(n=6$ for major CVAEs and $n=1$ for uncontrolled hypertension); moreover two patients required a dose reduction because 
of cardiovascular toxicity (Table S1 details the CFZ regimens of patients enrolled, Table S2 details the cumulative dosages of CFZ at first CVAE, available in Supplementary Materials).

Table 1. Baseline population characteristics.

\begin{tabular}{|c|c|}
\hline Characteristics & $\begin{array}{c}\text { Overall Population } \\
\text { No }=116\end{array}$ \\
\hline $\begin{array}{c}\text { General } \\
\text { Age, median (SD), years } \\
\text { Male sex, No. }(\%)\end{array}$ & $\begin{array}{c}64.53 \pm 8.42 \\
65(56)\end{array}$ \\
\hline $\begin{array}{c}\text { Individual CV risk factors, No (\%) } \\
\text { Tobacco use (prior/current) } \\
\text { Obesity (BMI } \geq 30) \\
\text { Known Arterial Hypertension } \\
\text { Diabetes } \\
\text { Chronic renal failure }(\mathrm{eGFR}<60 \mathrm{~mL} / \mathrm{m}) \\
\text { Ischemic heart disease } \\
\text { Previous episodes of atrial fibrillation } \\
\text { Dyslipidaemia } \\
\text { Previous stroke }\end{array}$ & $\begin{array}{c}58(50) \\
34(29.3) \\
48(41.4) \\
12(10.3) \\
22(19) \\
4(3.4) \\
2(1.7) \\
16(13.8) \\
0(0)\end{array}$ \\
\hline $\begin{array}{c}\text { Familiar CV risk factors, No }(\%) \\
\text { Ischemic heart disease } \\
\text { Stroke } \\
\text { Diabetes } \\
\text { Arterial Hypertension }\end{array}$ & $\begin{aligned} 26 & (22.4) \\
13 & (11.2) \\
6 & (5.2) \\
15 & (12.9)\end{aligned}$ \\
\hline $\begin{array}{c}\text { Anti-hypertensive drugs, No (\%) } \\
\text { Beta-blockers } \\
\text { ACE-inhibitors/angiotensin receptor blockers } \\
\text { Thiazide diuretics/Loop diuretics } \\
\text { Aldosterone receptor antagonists } \\
\text { Calcium channel blockers }\end{array}$ & $\begin{aligned} 26 & (22.4) \\
59 & (50.9) \\
25 & (21.6) \\
2 & (1.7) \\
25 & (21.6)\end{aligned}$ \\
\hline $\begin{array}{c}\text { Office BP values } \\
\text { SBP, mean (SD), mmHg } \\
\text { DBP, mean (SD), mmHg }\end{array}$ & $\begin{array}{c}129.4 \pm 17.8 \\
76.9 \pm 11.2\end{array}$ \\
\hline $\begin{array}{c}A B P M^{*} \\
\text { Daytime SBP, mean (SD), mmHg } \\
\text { Daytime DBP, mean (SD), mmHg } \\
24 \text { h SBP, mean (SD), mmHg } \\
24 \mathrm{~h} \mathrm{DBP}, \text { mean (SD), mmHg } \\
24 \mathrm{~h} \mathrm{MBP} \text { mean (SD), mmHg }\end{array}$ & $\begin{aligned} 125.0 & \pm 13.2 \\
75.1 & \pm 8.9 \\
120.9 & \pm 12.7 \\
71.7 & \pm 8.1 \\
88.5 & \pm 9.1\end{aligned}$ \\
\hline $\begin{array}{c}\text { HMOD, No (\%) } \\
\text { LVH } \\
\text { GLS value }{ }^{\dagger} \leq 20 \% \\
\text { PWV value }{ }^{\ddagger} \geq 9 \mathrm{~m} / \mathrm{s}\end{array}$ & $\begin{array}{l}24(20.7) \\
24(20.7) \\
31(26.7)\end{array}$ \\
\hline $\begin{array}{c}\text { Oncological History } \\
\text { Median MM disease duration, months } \\
\text { Carfilzomib line therapy, number } \\
\text { Previous therapies }{ }^{\prime}, \text { No. }(\%) \\
\text { Anthracyclines } \\
\text { Alkylating agents } \\
\text { Immunomodulating agents } \\
\text { Bortezomib }\end{array}$ & $\begin{array}{l}51.1 \\
3 \pm 1.6 \\
31(26.7) \\
91(78.4) \\
86(74.1) \\
97(83.6)\end{array}$ \\
\hline
\end{tabular}

Mean values estimated in: ${ }^{*} 107$ patients; ${ }^{\dagger} 115$ patients; ${ }^{\ddagger} 103$ patients. ${ }^{~ p a t i e n t s ~ w e r e ~ m o s t l y ~ t r e a t e d ~ w i t h ~}$ multiple therapies, hence total $\%$ might amount to $>100$. BMI $=$ body mass index; eGFR $=$ estimated glomerular filtration rate; $\mathrm{ABPM}=$ ambulatory blood pressure monitoring; $\mathrm{SBP}=$ systolic blood pressure; $\mathrm{DBP}=$ diastolic blood pressure; $\mathrm{MBP}=$ mean blood pressure; $\mathrm{LVH}=$ left ventricular hypertrophy (male $\mathrm{LV}$ mass $\geq 105 \mathrm{~g} / \mathrm{m}^{2}$; female LV mass $\geq 95 \mathrm{~g} / \mathrm{m}^{2}$ ); GLS = global longitudinal strain; $\mathrm{PWV}=$ pulse wave velocity; $\mathrm{HMOD}=$ hypertension mediated organ damage; $\mathrm{MM}=$ multiple myeloma. 


\subsection{Rates of CVAEs: All-Types, Major and Hypertension-Related CVAEs}

The subtypes of CVAE occurring during CFZ therapy are listed in Table 2. During a median follow-up of 10.8 months, 52 patients (44.9\%) experienced one or more CVAEs, with $30.9 \%$ grade 3 or greater in severity (CTCAE $\geq 3)$. 17 subjects $(14.7 \%)$ experienced major CVAEs, with four patients (23.5\%) experiencing more than one major CVAE. Arrhythmias $(41.2 \%)$, acute coronary syndromes $(23.5 \%)$ and post-infusion dyspnea (23.5\%), were the most commonly represented; one sudden cardiac death occurred after 22 months of CFZ therapy. 45 patients (38.7\%) experienced hypertension-related CVAEs, of which $72 \%$ were grade 1-2 in severity. 10 patients experienced both major and hypertensive CVAEs, with 13 CVAEs grade $\geq 3$ in severity. 27 patients (51.9\%) experienced CVAEs within the first three months of treatment.

Table 2. Lists of all-types of CVAE, major CVAEs and hypertension-related CVAEs.

\begin{tabular}{|c|c|c|c|}
\hline \multirow{2}{*}{ CVAE } & \multirow{2}{*}{$\begin{array}{c}\text { Patients } n=116 \\
\text { No. }(\%) *\end{array}$} & \multicolumn{2}{|c|}{ No. CVAEs } \\
\hline & & Grade 1-2 & Grade $\geq 3$ \\
\hline Major CVAEs & $17(14.7)$ & 12 & 10 \\
\hline ACS (STEMI) & $1(0.9)$ & 0 & 1 \\
\hline ACS (NSTEMI) & $3(2.6)$ & 0 & 3 \\
\hline Typical Chest pain & $3(2.6)$ & 3 & 0 \\
\hline Heart failure & $1(0.9)$ & 0 & 1 \\
\hline Dyspnoea post infusion & $4(3.5)$ & 2 & 2 \\
\hline Syncope/pre-syncope & $1(0.9)$ & 0 & 1 \\
\hline Arrhythmias & $7(6.0)$ & 6 & 1 \\
\hline Sudden cardiac death & $1(0.9)$ & NA & 1 \\
\hline Hypertension-related CVAEs & $45(38.7)$ & 75 & 29 \\
\hline New onset/worsened hypertension & $37(31.9)$ & 37 & 0 \\
\hline Masked hypertension & $4(3.5)$ & 4 & 0 \\
\hline White coat hypertension & $0(0)$ & 0 & 0 \\
\hline $\begin{array}{l}\text { Pre-infusion uncontrolled hypertension } \\
\text { [infusion limiting] }\end{array}$ & $11(9.5)$ & 6 & 9 \\
\hline $\begin{array}{l}\text { Pre-infusion uncontrolled hypertension } \\
\text { [not-infusion limiting] }\end{array}$ & $20(17.2)$ & 20 & 10 \\
\hline Post-infusion uncontrolled hypertension & $11(9.5)$ & 8 & 6 \\
\hline Symptomatic uncontrolled hypertension & $4(3.5)$ & 0 & 4 \\
\hline Hypertensive emergency & $0(0)$ & 0 & 0 \\
\hline All-type CVAEs & $52(44.9)$ & 87 & 39 \\
\hline Both major and hypertensive CVAEs & $10(8.6)$ & 26 & 13 \\
\hline
\end{tabular}

\subsection{Comparison between No-CVAEs and CVAEs Group: Baseline CV Parameters}

At baseline, patients experiencing CVAEs had higher BP values and higher indices of $B P$ variability at office and at ABPM, specifically higher systolic and diastolic office blood pressure values, higher daytime systolic BP and BP variability at ABPM. At echocardiographic evaluation, the CVAEs group had a significantly higher left ventricular mass and a reduction in GLS mean value, with no difference in LVEF. Furthermore, patients who experienced CVAEs had increased arterial stiffness at baseline, as demonstrated by the higher PWV value. No meaningful differences in age, sex, anthropometric variables, previous cardiovascular history, ECG, MM duration and previous oncological treatments (Anthracyclines, Alkylating agents, Immunomodulating agents, Bortezomib, All) were observed between the two groups. The baseline values that best predicted the incidence of CVAEs during CFZ therapy (variables made categorical using the Youden index and included in a logistic regression) were: office $\mathrm{SBP} \geq 131.5 \mathrm{mmHg}(\mathrm{OR}=5.41 ; p=0.001)$, $\mathrm{BP}$ variability $\geq 10$ at $\mathrm{ABPM}(\mathrm{OR}=5.071 ; p=0.002)$, presence of $\mathrm{LVH}$ at echocardiography 
$(\mathrm{OR}=3.056 ; p=0.021), \mathrm{PWV} \geq 8.75 \mathrm{~m} / \mathrm{s}(\mathrm{OR}=5.13 ; p=0.000)$. Table 3 details the comparison of the main baseline parameters between no-CVAEs and CVAEs group, and Table S3 details the remaining ones (available in Supplementary Materials).

Table 3. Comparison between no-CVAEs and CVAEs groups: main baseline parameters.

\begin{tabular}{|c|c|c|c|}
\hline Characteristics & $\begin{array}{c}\text { No CVAEs } \\
\text { No }=64(53.4 \%)\end{array}$ & $\begin{array}{c}\text { CVAEs } \\
\text { No }=52 \\
(46.6 \%)\end{array}$ & $p$ Value \\
\hline \multicolumn{4}{|l|}{ Demographic and clinical } \\
\hline Age, median (SD), years & $63.34 \pm 8.26$ & $66.00 \pm 8.46$ & 0.092 \\
\hline Male sex & $36(56.3)$ & $29(55.8)$ & 0.959 \\
\hline \multicolumn{4}{|l|}{ Individual CV risk factors, No (\%) } \\
\hline Tobacco use (past/current) & $29(45.3)$ & $29(55.8)$ & 0.263 \\
\hline Known Arterial Hypertension & $25(39.1)$ & $23(44.2)$ & 0.574 \\
\hline Diabetes & $7(10.9)$ & $5(9.6)$ & 0.816 \\
\hline Chronic renal failure $(\mathrm{eGFR}<60 \mathrm{~mL} / \mathrm{m})$ & $15(23.4)$ & $7(13.5)$ & 0.173 \\
\hline Coronary artery disease & $1(1.6)$ & $3(5.8)$ & 0.217 \\
\hline Previous Atrial Fibrillation & $2(3.1)$ & $0(0)$ & 0.501 \\
\hline Dyslipidaemia & $10(15.6)$ & $6(11.5)$ & 0.526 \\
\hline Previous stroke & $0(0)$ & $0(0)$ & NA \\
\hline Anti-hypertensive drugs $\geq 3$ & $3(4.7)$ & $5(9.6)$ & 0.298 \\
\hline \multicolumn{4}{|l|}{ Office BP values, mean (SD) } \\
\hline $\mathrm{SBP}, \mathrm{mmHg}$ & $124.71 \pm 17.25$ & $135.6 \pm 16.87$ & 0.002 \\
\hline $\mathrm{DBP}, \mathrm{mmHg}$ & $74.73 \pm 12.39$ & $79.35 \pm 9.12$ & 0.024 \\
\hline HR, bpm & $77.22 \pm 13.05$ & $76.29 \pm 13.49$ & 0.720 \\
\hline \multicolumn{4}{|l|}{$A B P M$ * } \\
\hline Daytime SBP, mean (SD), $\mathrm{mmHg}$ & $122.98 \pm 13.88$ & $127.39 \pm 12.15$ & 0.008 \\
\hline Daytime DBP, mean (SD), $\mathrm{mmHg}$ & $73.95 \pm 9.40$ & $76.37 \pm 8.31$ & 0.534 \\
\hline Daytime SD, mean (SD) & $10.81 \pm 3.80$ & $15.14 \pm 11.62$ & 0.009 \\
\hline $24 \mathrm{~h} \mathrm{SBP}$, mean (SD), mmHg & $119.24 \pm 13.48$ & $122.94 \pm 11.63$ & 0.131 \\
\hline $24 \mathrm{~h}$ DBP, mean (SD), mmHg & $70.76 \pm 8.38$ & $72.73 \pm 7.73$ & 0.208 \\
\hline $24 \mathrm{~h} \mathrm{MBP}$, mean (SD), mmHg & $87.69 \pm 9.54$ & $89.39 \pm 8.57$ & 0.336 \\
\hline $24 \mathrm{~h} \mathrm{SD}$, mean (SD) & $12.43 \pm 3.82$ & $15.11 \pm 4.64$ & 0.002 \\
\hline Night time SD, mean (SD) & $8.45 \pm 2.82$ & $10.22 \pm 4.02$ & 0.011 \\
\hline Dipping $<10 \%$, No. $(\%)$ & $25(43.9)$ & $13(28.9)$ & 0.120 \\
\hline Blood pressure variability, No. (\%) & $8.10 \pm 2.47$ & $10.31 \pm 4.08$ & 0.001 \\
\hline \multicolumn{4}{|l|}{ Echocardiography ${ }^{\dagger}$} \\
\hline LVMi, mean (SD), $\mathrm{g} / \mathrm{m}^{2}$ & $85.30 \pm 19.72$ & $95.14 \pm 21.75$ & 0.013 \\
\hline LVEF, mean (SD), \%* & $63.03 \pm 6.56$ & $61.96 \pm 7.13$ & 0.414 \\
\hline GLS, mean (SD), \%* & $-22.37 \pm 2.56$ & $-21.3 \pm 2.46$ & 0.029 \\
\hline Diastolic dysfunction, No. (\%) & $1(1.6)$ & $0(0)$ & 0.362 \\
\hline \multicolumn{4}{|l|}{ Arterial Stiffness $\ddagger$} \\
\hline PWV value, mean (SD), $\mathrm{m} / \mathrm{s}$ & $7.41 \pm 1.63$ & $8.55 \pm 1.855$ & 0.002 \\
\hline
\end{tabular}

Mean values estimated in: ${ }^{*} 107$ patients; +115 patients; $\ddagger 103$ patients. $p$ value $<0.05$ highlighted in bold format. $\mathrm{BMI}=$ body mass index; $\mathrm{eGFR}=$ estimated glomerular filtration rate; $\mathrm{ABPM}=$ ambulatory blood pressure monitoring; $\mathrm{SBP}=$ systolic blood pressure; $\mathrm{DBP}=$ diastolic blood pressure; $\mathrm{HR}=$ heart rate; $\mathrm{SD}=$ standard deviation; $\mathrm{MBP}=$ mean blood pressure; $\mathrm{LVMi}=$ left ventricular mass indexed to body surface area; $\mathrm{LVEF}=$ left ventricular ejection fraction; GLS = global longitudinal strain; $\mathrm{PWV}=$ pulse wave velocity.

\subsection{CFZ CVAEs Risk Score}

Firstly, we performed a penalized regression including variables which showed a $p$ value $<0.005$ at the univariate logistic regression together with those for which a predictive value was to be expected based on current literature (age, sex, diabetes, ischemic heart disease, chronic kidney disease, office SBP, BPV, PWV, LVEF, LVH and GLS). According to the CHARMS Recommendations [15], to comply with an event per variable (EPV- number of events / number of variables) $>10$, we performed a variable selection by penalized logistic regression (elastic net regression) fixing a maximum of five output variables with non-zero 
coefficient. SBP office, BPV, PWV, LVH and GLS had their coefficients not forced to zero, therefore they were included in the second penalized regression. Coefficients extracted from this model were used to calculate the CFZ CVAEs Risk Score equation:

CFZ CVAEs Risk Score $=-2.84666907+(0.08244733 \cdot \mathrm{BPV})+(0.17791129 \cdot \mathrm{PWV})+$ $(0.57680056 \cdot \mathrm{LVH})+(-0.06201887 \cdot \mathrm{GLS})+(0.01282460 \cdot$ office SBP $)$

Therefore, the predicted risk of event was:

$$
\text { Score }=\frac{e^{\text {Score Risk }}}{1+e^{\text {Score Risk }}}
$$

We pre-determined the sensibility value to be $\geq 90 \%$ and obtained a risk-score cut-off of $32 \%$ to divide the study sample into a low- and a high-risk group for CVAEs (respectively 30 and 86 patients). The resulting negative predictive value for CVAEs in the high-risk group was $90 \%$ (with a specificity of $42 \%$ and AUC of 0.76 ). Figure 2 shows the probability of incurring CVAEs to increasing of CFZ CVAEs Risk. 3 patients in the low-risk group and 49 patients in the high-risk group experienced CVAEs. The validation, in absence of another different sample, was conducted through the method the internal validation, using the bootstrap methodology (Figures 3 and 4). Mean $C$ statistic was $0.76 \pm 0.06$ : the smooth calibration curve fit the perfect condition (observed $=$ expected), with a calibration slope close to $1(1.0 \pm 0.1)$ and a calibration intercept close to $0(0.0 \pm 0.1)$, as shown in Figure 5.

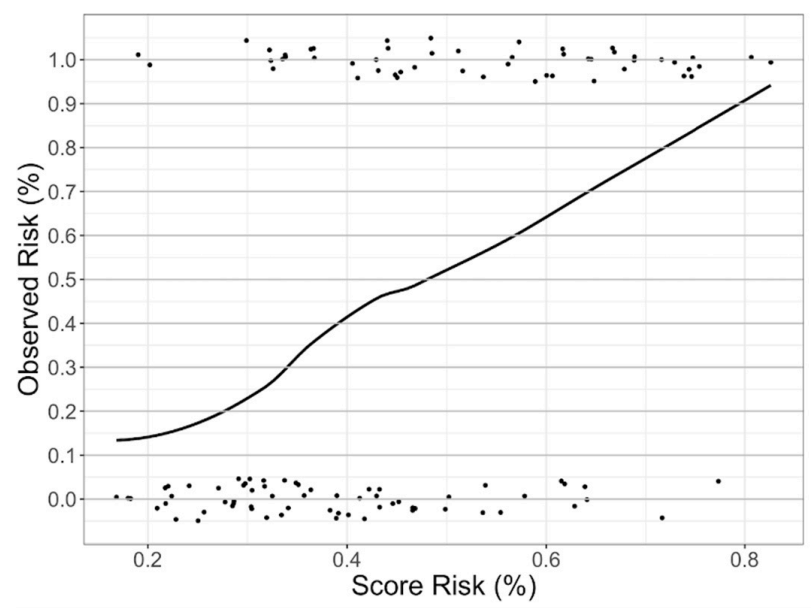

Figure 2. Probability of incurring CVAEs related to increasing of carfilzomib (CFZ) CVAEs Risk Score. Vertical axis = observed CVAE events (\%); Horizontal axis = predicted CFZ CVAE risk (\%). The upper points represent patients who experienced CVAEs; the lower points represent patients who did not experienced CVAEs.

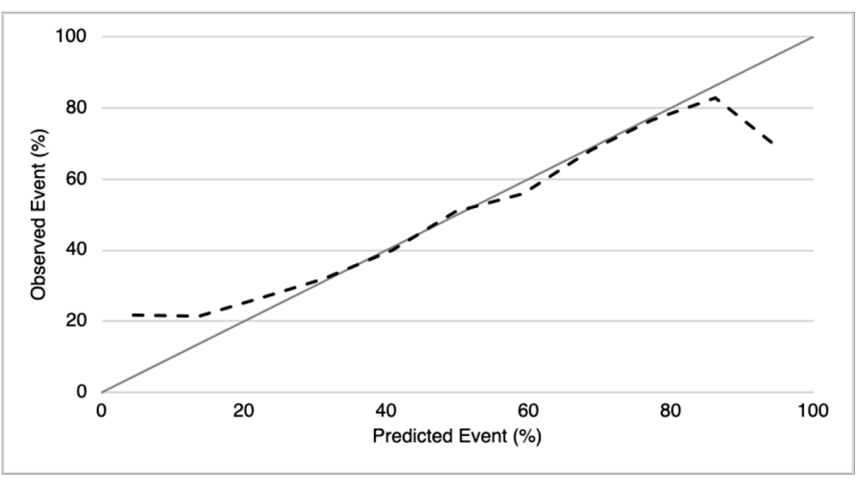

Figure 3. Smooth Calibration plot for the validation of CFZ CVAEs Risk Score. Comparison between observed and predicted CVAEs based on bootstrap. The grey line represents the perfect condition in which the observed CVAEs are equal to predicted CVAEs; the black line represents the CVAEs observed in our population. 


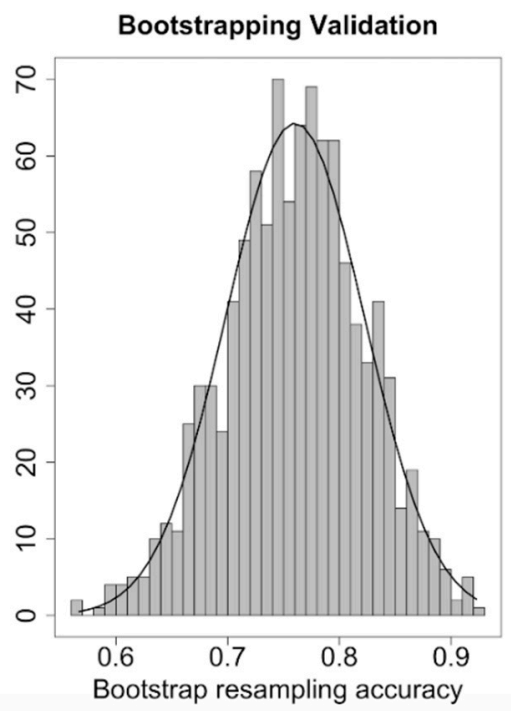

Figure 4. CFZ CVAEs Risk Score Discrimination plot.
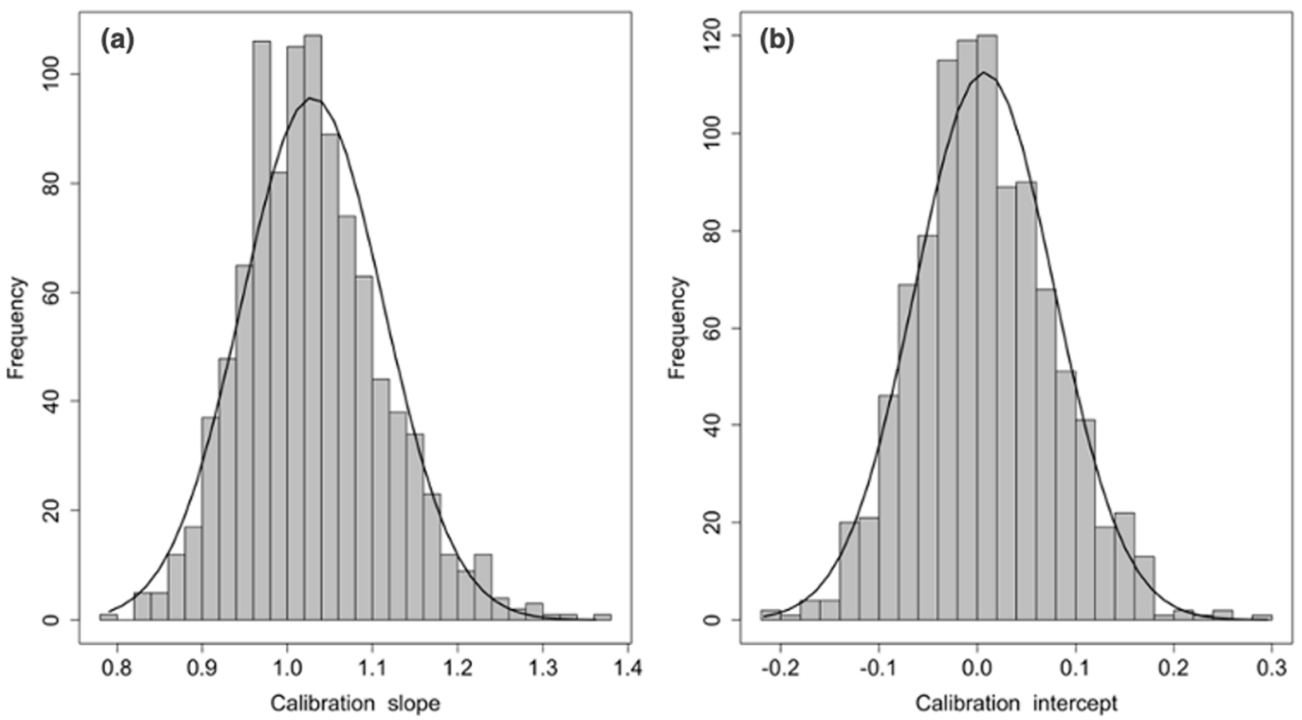

Figure 5. CFZ CVAEs Risk Score Calibration: (a) calibration slope and (b) calibration intercepts.

\section{Discussion}

Carfilzomib therapy is closely related to high risk of CVAEs in MM patients, with a large variability in type and severity of events. It is clear that a cardiovascular baseline assessment is pivotal in MM patients undergoing drugs with a potentially cardio-toxic profile, such as CFZ. In this regard, previous cardiovascular risk assessment protocols have been proposed in cancer patients undergoing cardio-toxic treatments [20-22]; however, no prospective studies have validated their effectiveness in MM. Besides this, the same ESH Systematic Coronary Risk Evaluation (SCORE) [23] might be inadequate to estimate the global CV risk in this complex category of patients. In this study, MM patients scheduled for CFZ therapy underwent a multisystemic assessment according to the Consensus Paper of the European Hematology Association joint with thevEuropean Myeloma Network [8]. By applying this protocol, at baseline, MM patients showed a high CV risk profile, as demonstrated by the large prevalence of uncontrolled blood pressure at office and/or ABPM and by the high proportion of asymptomatic target organ damage. However, currently there is limited evidence regarding predictors of CVAEs with conflicting results, due to the lack of prospective studies [22]. Among baseline parameters, we investigated which variables before starting CFZ could predict the risk of CVAEs occurring. 
Through consecutive penalized regressions, we identified five independent predictors of CVAEs which were included in the model 'the CVAEs risk score': (1) office SBP value, (2) BPV value at ABPM, (3) GLS value, (4) presence/absence of LVH at echocardiography and (5) PWV value at arterial stiffness estimation. In the score, two variables referred to blood pressure control: SBP at office measurements and BPV at out-of-office recording. The systematic assessment of ABPM confirmed in MM patients the association between a high BPV, cardiovascular events and disease mortality [24,25], previously described in hypertensive patients. Regarding the GLS, we found that its reduction at baseline was a leading risk factor for CVAEs, as opposed to LVEF, suggesting the relevant prognostic value of functional LV baseline evaluation, even if the GLS assessment is not systematically recommended [22]. Hence, the GLS confirmed its superiority in detection of early subclinical LV myocardial injury, as previously demonstrated in the general population $[26,27]$. Concerning the PWV, in our study the increase of arterial stiffness, was an independent predictor of CVAEs [28]. Although it is a known CV risk factor, recommended as a part of hypertensive patients, until now its role in cancer patients was not well established. These five parameters were included in the model in which the CV risk depends on a complex interaction between the predictors' value that relate to one another. Using the CVAEs risk score, patients were distinguished into a low and a high-risk group of incurring a cardiovascular event (major and/or hypertensive). During the first visit, the application of this score by the clinician, might be a useful tool to identify patients at high-risk of incurring a CVAE, and consequently discontinuing CFZ therapy, who might benefit from specific risk mitigation strategies and follow-up. Regarding the comparative analysis between no-CVAEs and CVAEs group, we described differences in daytime SBP, mean $24 \mathrm{~h}$ $\mathrm{SD}$, left ventricular mass and others; however, the overlap between ranges of many of these does not allow their use in clinical practice at this moment.

Regarding the analysis of CVAEs, we fully described major and hypertension-related CVAEs, proving that up to $45 \%$ of patients undergoing CFZ therapy experienced CVAEs. This data is consistent with the PROTECT study [5] which showed an incidence of 50.7\% cardiovascular events, but in contrast with other clinical trials, in which the rate of CVAEs ranged from $18 \%$ to $22 \%$ [7,29-31]. These results might be explained by the real-life design of our study and of Cornell's report, including patients with comorbidities of varying severity, and by the specific focus on hypertensive CVAEs. Indeed, we observed a greater incidence of hypertensive CVAEs than previously reported: up to $39 \%$ of patients experienced one or more hypertensive events. Conversely to Cornell at al., this result was due to the choice of also including 1-2 degree hypertension events in order to be more sensible in identifying patients with a potential risk of developing aHMOD and future CV events. However, despite the high rate of CVAEs observed, only few patients required a discontinuation of CFZ therapy or a reduction of drug dosage. In fact, the majority of CVAE events were transient with low severity; in particular, among hypertensive events, only one patient discontinued CFZ for uncontrolled blood pressure. For all the remaining cases, patients continued CFZ therapy after an intensification of the hypertensive treatment. Despite the accurate recognition and the early-stage treatment of patients with arterial hypertension, a high rate of hypertensive events was observed, suggesting that the optimization of blood pressure values might be not sufficient. Periodic monitoring of BP values and frequent rechecking of adequate blood pressure control seems to be essential during CFZ therapy. In summary, based on the results, the application in clinical practice of the standardized protocol of the EMN to MM patients scheduled for CFZ therapy allows the correct estimation of the CV risk profile. The evaluation of the blood pressure (office and out of office), echocardiography (including LVH and GLS) and arterial stiffness (through the PWV) are useful to calculate the 'CVAEs risk score' in order to identify patients at higher risk of CVAEs during CFZ therapy, and needing a tailored follow-up.

Our study has several limitations. Firstly, the relatively small sample of the cohort without a control arm did not allow the detection of potential confounding factors. Due to the lack of sufficient data on laboratory biomarkers (NT-proBNP and cardiac troponins), 
it was not possible to test their prognostic significance. Regarding the tests done during the comprehensive baseline evaluation, the ABPM, the GLS and PWV assessment were not available for all patients due to technical reasons. Furthermore, the evaluation of arterial stiffness through the PWV assessment is not available in all Centers and this could limit the clinical applicability of the score. Concerning the consistent incidence of hypertensive CVAEs observed, this may have been affected both by the optimization of anti-hypertensive treatment prior to CFZ therapy and by the accurate detection of all hypertensive events during treatment, due to the specialization of our setting (Hypertension Unit). Another limitation was the absence of standardized dosing of the CFZ regimen that might explain the absence of relationship between dosing and CVAEs. Concerning the risk score, we underline two limitations: the model had to be internally validated due to the lack of an independent data sample and, in order to prevent reduction in the patient cohort, we computed missing data with a multiple imputation system. Due to these limitations, further studies are needed to validate the model on an independent larger sample.

\section{Conclusions}

CVAEs are frequent during CFZ therapy in MM and a specific management protocol is crucial. The application of the standardized protocol proposed by the European Myeloma Network and the use of the 'CFZ CVAEs risk score' proved to be effective in estimating the baseline risk for CVAEs during CFZ therapy, allowing for a patient-tailored follow-up and for the adoption of effective risk mitigation strategies.

Supplementary Materials: The following are available online at https: / www.mdpi.com/article/ 10.3390/cancers13071631/s1, Table S1: Carfilzomib-based chemotherapeutic regimens, Table S2: Carfilzomib cumulative dose and time at the first all-type CVAE, major CVAE, hypertension-related CVAE and at the end of the planning therapy, Table S3: Comparison between no-CVAEs and CVAEs groups: additional baseline parameters.

Author Contributions: Conceptualization, A.A. and A.M.; methodology, A.A., G.M., and F.V. (Fabrizio Vallelonga); software, L.A. and C.G.; validation, L.A.; formal analysis, A.A., L.A., and C.G.; data curation, A.A, G.M., C.C., M.C. (Marco Cesareo ), G.B., D.L. and G.C.; writing-original draft preparation, A.A.; writing-review and editing, A.M., M.C. (Michele Covella), S.B., F.G., M.S., F.R., F.V. (Franco Veglio); supervision, A.M.; project administration, A.M and F.V. (Franco Veglio). All authors have read and agreed to the published version of the manuscript.

Funding: This research received no external funding.

Institutional Review Board Statement: The study was conducted according to the guidelines of the Declaration of Helsinki and approved by the Ethics Committee of the 'Città della Salute e della Scienza' Hospital of Turin (Protocol Number 0038655).

Informed Consent Statement: Informed consent was obtained from all subjects involved in the study.

Data Availability Statement: Not applicable.

Acknowledgments: Ambra Fabbri, M.D. for technical support.

Conflicts of Interest: Alberto Milan received honoraria for advisory board from Amgen and Janssen. Sara Bringhen received honoraria from Bristol-Myers Squibb, Celgene, Amgen and Janssen; advisory boards for Amgen, Karyopharm, Janssen and Celgene; consultancy fees from Takeda and Janssen. Francesca Gay received honoraria from Amgen, Janseen, Celgene, BMS, Takeda, Abbvie; advisory boards for Amgen, Janseen, Celgene, BMS, Takeda, Abbvie; advisory adaptive for Roche Oncopeptides. The other authors declare no conflict of interest. The funders had no role in the design of the study; in the collection, analyses, or interpretation of data; in the writing of the manuscript, or in the decision to publish the results. 


\begin{abstract}
Appendix A
Appendix A.1 Major Cardiovascular Adverse Events Definitions

Acute coronary syndromes, distinguished in ST and no-ST myocardium infarction, were diagnosed in presence of increase/decrease of a cardiac biomarker (with at least one value above the 99th percentile) and at least one of the following: symptoms, ischemic ECG changes, echocardiographic findings [32]. Heart failure was demonstrated by the presence of at least two of the three following items: symptoms and/or clinical signs, echocardiographic findings, elevated levels of natriuretic peptides [33]. Dyspnea related to CFZ-infusion was defined by a new onset of dyspnea within three days post CFZinfusion, without clinical or instrumental signs of heart failure or pulmonary hypertension. Arrhythmias included atrial fibrillation or flutter, atrial bigeminy, ventricular tachycardia, ventricular bigeminy/trigeminy, and grade $\geq 2$ of atrioventricular block. Typical chest pain was defined by the presence of chest pain with features suggestive of ischemic cardiac origin (duration, irradiation, onset, etc), and for this reason subjected to clinical and instrumental assessments (ECG, cardiac troponins and eventually echocardiography) with negative results. Syncope was defined by a spontaneous loss of consciousness, presyncope by dizziness and feeling faint (with or without hypotension) with evidence of cardiac origin at clinical and instrumental evaluations. Sudden cardiac death was defined by unexpected death during CFZ therapy without other proven causes.
\end{abstract}

\title{
Appendix A.2 Arterial Hypertension Cardiovascular Adverse Events Definitions
}

New diagnosis of arterial hypertension was defined in presence of blood pressure values $\geq 140 / 90 \mathrm{mmHg}$ at office or out-of-office BP measurements, in subjects prior normotensive (9); the worsening of known arterial hypertension was defined as increased BP values $\geq 140 / 90 \mathrm{mmHg}$ requiring additional anti-hypertensive treatment in subject with known arterial hypertension. CFZ pre-infusion uncontrolled hypertension was defined by office $B P$ values $\geq 140 / 90 \mathrm{mmHg}$ within 30 minutes prior $C F Z$ infusion, distinguished by limiting or permissive CFZ infusion. CFZ post-infusion uncontrolled hypertension was defined by office $\mathrm{BP}$ values $\geq 140 / 90 \mathrm{mmHg}$ within 30 minutes from CFZ infusion. Masked hypertension was defined by the presence of arterial hypertension at ABPM when office BP measurements were normal; white coat hypertension was defined by normal ABPM values but elevated office BP [9]. Symptomatic uncontrolled blood pressure was demonstrated by symptomatic cases of $\mathrm{BP}>180 / 110 \mathrm{mmHg}$ subjected to further assessment that excluded target acute organ damage; hypertensive emergency was defined by symptomatic BP $>180 / 110 \mathrm{mmHg}$, with evidence of target acute organ damage at further assessment tests [9].

\section{References}

1. Siegel, D.S.; Martin, T.; Wang, M.; Vij, R.; Jakubowiak, A.J.; Lonial, S.; Trudel, S.; Kukreti, V.; Bahlis, N.; Alsina, M.; et al. A phase 2 study of single-agent carfilzomib (PX-171-003-A1) in patients with relapsed and refractory multiple myeloma. Blood 2012, 120, 2817-2825. [CrossRef] [PubMed]

2. Stewart, A.K.; Rajkumar, S.V.; Dimopoulos, M.A.; Masszi, T.; Špička, I.; Oriol, A.; Hájek, R.; Rosiñol, L.; Siegel, D.S.; Mihaylov, G.G.; et al. Carfilzomib, Lenalidomide, and Dexamethasone for Relapsed Multiple Myeloma. N. Engl. J. Med. 2015, 372, 142-152. [CrossRef] [PubMed]

3. A Dimopoulos, M.; Moreau, P.; Palumbo, A.; Joshua, D.; Pour, L.; Hájek, R.; Facon, T.; Ludwig, H.; Oriol, A.; Goldschmidt, H.; et al. Carfilzomib and dexamethasone versus bortezomib and dexamethasone for patients with relapsed or refractory multiple myeloma (ENDEAVOR): A randomised, phase 3, open-label, multicentre study. Lancet Oncol. 2016, 17, 27-38. [CrossRef]

4. Hájek, R.; Masszi, T.; Petrucci, M.T.; Palumbo, A.; Rosiñol, L.; Nagler, A.; Yong, K.L.; Oriol, A.; Minarik, J.; Pour, L.; et al. A randomized phase III study of carfilzomib vs low-dose corticosteroids with optional cyclophosphamide in relapsed and refractory multiple myeloma (FOCUS). Leukemia 2017, 31, 107-114. [CrossRef]

5. Cornell, R.F.; Ky, B.; Weiss, B.M.; Dahm, C.N.; Gupta, D.K.; Du, L.; Carver, J.R.; Cohen, A.D.; Engelhardt, B.G.; Garfall, A.L.; et al Prospective Study of Cardiac Events During Proteasome Inhibitor Therapy for Relapsed Multiple Myeloma. J. Clin. Oncol. 2019, 37, 1946-1955. [CrossRef] [PubMed] 
6. Chen-Scarabelli, C.; Corsetti, G.; Pasini, E.; Dioguardi, F.S.; Sahni, G.; Narula, J.; Gavazzoni, M.; Patel, H.; Saravolatz, L.; Knight, R.; et al. Spasmogenic Effects of the Proteasome Inhibitor Carfilzomib on Coronary Resistance, Vascular Tone and Reactivity. EBio Med. 2017, 21, 206-212. [CrossRef]

7. Waxman, A.J.; Clasen, S.; Hwang, W.-T.; Garfall, A.; Vogl, D.T.; Carver, J.; O’Quinn, R.; Cohen, A.D.; Stadtmauer, E.A.; Ky, B.; et al. Carfilzomib-Associated Cardiovascular Adverse Events. JAMA Oncol. 2018, 4, e174519. [CrossRef]

8. Bringhen, S.; Milan, A.; D'Agostino, M.; Ferri, C.; Wäsch, R.; Gay, F.; LaRocca, A.; Offidani, M.; Zweegman, S.; Terpos, E.; et al. Prevention, monitoring and treatment of cardiovascular adverse events in myeloma patients receiving carfilzomib A consensus paper by the European Myeloma Network and the Italian Society of Arterial Hypertension. J. Intern. Med. 2019, 286, 63-74. [CrossRef]

9. Williams, B.; Mancia, G.; Spiering, W.; Rosei, E.A.; Azizi, M.; Burnier, M.; Clement, D.L.; Coca, A.; de Simone, G.; Dominiczak, A.F.; et al. 2018 ESC/ESH Guidelines for the management of arterial hypertension. Eur. Heart J. 2018, 39, 3021-3104. [CrossRef]

10. Lang, R.M.; Badano, L.P.; Mor-Avi, V.; Afilalo, J.; Armstrong, A.; Ernande, L.; Flachskampf, F.A.; Foster, E.; Goldstein, S.A.; Kuznetsova, T.; et al. Recommendations for cardiac chamber quantification by echocardiography in adults: An update from the American society of echocardiography and the European association of cardiovascular imaging. Eur. Heart J. Cardiovasc. Imaging 2015, 16, 233-271. [CrossRef]

11. Nagueh, S.F.; Smiseth, O.A.; Appleton, C.P.; Byrd, B.F.; Dokainish, H.; Edvardsen, T.; Flachskampf, F.A.; Gillebert, T.C.; Klein, A.L.; Lancellotti, P.; et al. Recommendations for the Evaluation of Left Ventricular Diastolic Function by Echocardiography: An Update from the American Society of Echocardiography and the European Association of Cardiovascular Imaging. Eur. Hear. J. Cardiovasc. Imaging 2016, 17, 1321-1360. [CrossRef] [PubMed]

12. Voigt, J.-U.; Pedrizzetti, G.; Lysyansky, P.; Marwick, T.H.; Houle, H.; Baumann, R.; Pedri, S.; Ito, Y.; Abe, Y.; Metz, S.; et al. Definitions for a Common Standard for 2D Speckle Tracking Echocardiography: Consensus Document of the EACVI/ASE/Industry Task Force to Standardize Deformation Imaging. J. Am. Soc. Echocardiogr. 2015, 28, 183-193. [CrossRef] [PubMed]

13. Van Bortel, L.M.; Laurent, S.; Boutouyrie, P.; Chowienczyk, P.; Cruickshank, J.; De Backer, T.; Filipovsky, J.; Huybrechts, S.; Mattace-Raso, F.U.; Protogerou, A.D.; et al. Expert consensus document on the measurement of aortic stiffness in daily practice using carotid-femoral pulse wave velocity. J. Hypertens. 2012, 30, 445-448. [CrossRef] [PubMed]

14. Cancer Therapy Evaluation Program (CTEP): Common Terminology Criteria for Adverse Events (CTCAE) v.5.0. Cancer Ther. Eval. Progr. 2020. Update. Available online: https://ctep.cancer.gov/protocoldevelopment/electronic_applications/docs/ CTCAE_v5_Quick_Reference_5x7.pdf (accessed on 1 March 2020).

15. Moons, K.G.M.; De Groot, J.A.H.; Bouwmeester, W.; Vergouwe, Y.; Mallett, S.; Altman, D.G.; Reitsma, J.B.; Collins, G.S. Critical Appraisal and Data Extraction for Systematic Reviews of Prediction Modelling Studies: The CHARMS Checklist. PLoS Med. 2014, 11, e1001744. [CrossRef]

16. Collins, G.S.; Reitsma, J.B.; Altman, D.G.; Moons, K.G.M. Transparent reporting of a multivariable prediction model for individual prognosis or diagnosis (TRIPOD): The TRIPOD statement. BMJ 2014, 350, g7594. [CrossRef]

17. Marshall, A.; Altman, D.G.; Royston, P.; Holder, R.L. Comparison of techniques for handling missing covariate data within prognostic modelling studies: A simulation study. BMC Med. Res. Methodol. 2010, 10, 7. [CrossRef] [PubMed]

18. Pavlou, M.; Ambler, G.; Seaman, S.R.; Guttmann, O.; Elliott, P.; King, M.; Omar, R.Z. How to develop a more accurate risk prediction model when there are few events. BMJ 2015, 351, h3868. [CrossRef] [PubMed]

19. Moreau, P.; Miguel, J.S.; Sonneveld, P.; Mateos, M.V.; Zamagni, E.; Avet-Loiseau, H.; Hajek, R.; Dimopoulos, M.A.; Ludwig, H.; Einsele, H.; et al. Multiple myeloma: ESMO Clinical Practice Guidelines for diagnosis, treatment and follow-up. Ann. Oncol. 2017, 28, iv52-iv61. [CrossRef]

20. Zamorano, J.L.; Lancellotti, P.; Rodriguez Muñoz, D.; Aboyans, V.; Asteggiano, R.; Galderisi, M.; Habib, G.; Lenihan, D.J.; Lip, G.Y.H.; Lyon, A.R.; et al. 2016 ESC Position Paper on cancer treatments and cardiovascular toxicity developed under the auspices of the ESC Committee for Practice Guidelines. Eur. Heart J. 2016, 37, 2768-2801. [CrossRef]

21. Plana, J.C.; Galderisi, M.; Barac, A.; Ewer, M.S.; Ky, B.; Scherrer-Crosbie, M.; Ganame, J.; Sebag, I.A.; Agler, D.A.; Badano, L.P.; et al. Expert Consensus for Multimodality Imaging Evaluation of Adult Patients during and after Cancer Therapy: A Report from the American Society of Echocardiography and the European Association of Cardiovascular Imaging. J. Am. Soc. Echocardiogr. 2014, 27, 911-939. [CrossRef]

22. Lyon, A.R.; Dent, S.; Stanway, S.; Earl, H.; Brezden-Masley, C.; Cohen-Solal, A.; Tocchetti, C.G.; Moslehi, J.J.; Groarke, J.D.; Bergler-Klein, J.; et al. Baseline cardiovascular risk assessment in cancer patients scheduled to receive cardiotoxic cancer therapies: A position statement and new risk assessment tools from the $\mathrm{C}$ ardio- $\mathrm{O}$ ncology $\mathrm{S}$ tudy $\mathrm{G}$ roup of the $\mathrm{H}$ eart $\mathrm{F}$ ailure $\mathrm{A}$ ssociation of the E uropean $\mathrm{S}$ ociety of $\mathrm{C}$ ardiology in collaboration with the I nternational C ardio- O ncology S ociety. Eur. J. Hear. Fail. 2020, 22, 1945-1960. [CrossRef]

23. Conroy, R.M.; Pyörälä, K.; Fitzgerald, A.P.; Sans, S.; Menotti, A.; De Backer, G.; De Bacquer, D.; Ducimetière, P.; Jousilahti, P.; Keil, U.; et al. Estimation of ten-year risk of fatal cardiovascular disease in Europe: The SCORE project. Eur. Heart J. 2003, 24, 987-1003. [CrossRef]

24. Stevens, S.L.; Wood, S.; Koshiaris, C.; Law, K.; Glasziou, P.; Stevens, R.J.; McManus, R.J. Blood pressure variability and cardiovascular disease: Systematic review and meta-analysis. BMJ 2016, 354, i4098. [CrossRef] 
25. Parati, G.; Stergiou, G.S.; Dolan, E.; Bilo, G. Blood pressure variability: Clinical relevance and application. J. Clin. Hypertens. 2018, 20, 1133-1137. [CrossRef] [PubMed]

26. Rhea, I.B.; Uppuluri, S.; Sawada, S.; Schneider, B.P.; Feigenbaum, H. Incremental Prognostic Value of Echocardiographic Strain and Its Association With Mortality in Cancer Patients. J. Am. Soc. Echocardiogr. 2015, 28, 667-673. [CrossRef]

27. Kuznetsova, T.; Cauwenberghs, N.; Knez, J.; Yang, W.-Y.; Herbots, L.; D’Hooge, J.; Haddad, F.; Thijs, L.; Voigt, J.-U.; Staessen, J.A. Additive Prognostic Value of Left Ventricular Systolic Dysfunction in a Population-Based Cohort. Circ. Cardiovasc. Imaging 2016, 9. [CrossRef]

28. Bruno, G.; Bringhen, S.; Maffei, I.; Iannaccone, A.; Crea, T.; Ravera, A.; Astarita, A.; Vallelonga, F.; Salvini, M.; Gay, F.; et al. Cardiovascular Organ Damage and Blood Pressure Levels Predict Adverse Events in Multiple Myeloma Patients Undergoing Carfilzomib Therapy. Cancers 2019, 11, 622. [CrossRef] [PubMed]

29. Atrash, S.; Tullos, A.; Panozzo, S.; Bhutani, M.S.; Van Rhee, F.; Barlogie, B.; Usmani, S.Z. Cardiac complications in relapsed and refractory multiple myeloma patients treated with carfilzomib. Blood Cancer J. 2015, 5, e272. [CrossRef] [PubMed]

30. Chari, A.; Hajje, D. Case series discussion of cardiac and vascular events following carfilzomib treatment: Possible mechanism, screening, and monitoring. BMC Cancer 2014, 14, 915. [CrossRef]

31. Danhof, S.; Schreder, M.; Rasche, L.; Strifler, S.; Einsele, H.; Knop, S. 'Real-life' experience of preapproval carfilzomib-based therapy in myeloma-Analysis of cardiac toxicity and predisposing factors. Eur. J. Haematol. 2015, 97, 25-32. [CrossRef] [PubMed]

32. Ibanez, B.; James, S.; Agewall, S.; Antunes, M.J.; Bucciarelli-Ducci, C.; Bueno, H.; Caforio, A.L.P.; Crea, F.; A Goudevenos, J.; Halvorsen, S.; et al. 2017 ESC Guidelines for the management of acute myocardial infarction in patients presenting with STsegment elevation: The Task Force for the management of acute myocardial infarction in patients presenting with ST-segment elevation of the European Society of Cardiology (ESC). Eur. Heart J. 2017, 39, 119-177. [CrossRef]

33. Ponikowski, P.; Voors, A.A.; Anker, S.D.; Bueno, H.; Cleland, J.G.F.; Coats, A.J.S.; Falk, V.; González-Juanatey, J.R.; Harjola, V.-P.; Jankowska, E.A.; et al. 2016 ESC Guidelines for the diagnosis and treatment of acute and chronic heart failure: The Task Force for the diagnosis and treatment of acute and chronic heart failure of the European Society of Cardiology (ESC)Developed with the special contribution of the Heart Failure Association (HFA) of the ESC. Eur. Heart J. 2016, 37, 2129-2200. [CrossRef] [PubMed] 